Le rayonnement $X$ intense

des sources synchrotrons

modernes s'est imposé comme

un outil incontournable

pour les études in situ sous

conditions extrêmes de

pression et de température.

Deux études récentes

ont été sélectionnées

pour illustrer le potentiel

de ce type d'expérience.

Le premier exemple réside

dans la détermination

précise de la courbe

de fusion du tantale par

diffraction X. Le second

exemple concerne l'étude

de la densité du magma

lunaire par absorption $X$;

ces mesures permettent

d'expliquer simplement

l'absence d'activité volcanique

à la surface de la Lune.

\title{
Le rayonnement synchrotron: un outil de pointe pour la science sous conditions extrêmes de pression et de température
}

Mohamed Mezouar(i) (mezouar@esrf.fr), Agnès Dewaele ${ }^{(2)}$, Nicolas Guignot ${ }^{(3)}$

et Paul Loubeyre(2)

(1) European Synchrotron Radiation Facility, BP 220, 38043 Grenoble Cedex 9

(2) CEA / DAM Île-de-France, Bruyères-le-Châtel, 91297 Arpajon Cedex

(3) Synchrotron SOLEIL, L'Orme des Merisiers, 91192 Gif-sur-Yvette

Les études in situ par diffusion ou absorption de rayons $\mathrm{X}$ sous conditions extrêmes de pression et de température sont très délicates, en raison de la nécessité de travailler sur des échantillons de très faible volume (typiquement de l'ordre de $10^{-6} \mathrm{~mm}^{3}$ pour une pression de $100 \mathrm{GPa}$ ) et du caractère fortement absorbant des enceintes hautes pressions. Ceci explique l'émergence tardive de ce champ de recherche, pour lequel l'utilisation de sources conventionnelles de rayons $\mathrm{X}$ (anode tournante) est très limitée. Dès l'apparition des sources intenses, de haute énergie $(\mathrm{E}>30 \mathrm{keV})$ et faiblement divergentes, de rayons $\mathrm{X}$ que sont les synchrotrons dits "de troisième génération ", des progrès remarquables ont été réalisés dans ce domaine de recherche. Cela tient aux propriétés exceptionnelles de ces sources, qui permettent de sonder précisément la matière confinée à l'extrême dans des cavités à très hautes pressions.

Parallèlement, la technologie des hautes pressions a évolué de façon remarquable et a fourni des outils parfaitement adaptés à ces nouvelles sources de rayons X. Des découvertes spectaculaires ont été réalisées dans des domaines aussi variés que les sciences de la Terre, la physique, la chimie ou la biologie. On peut, par exemple, citer la découverte par diffraction de rayons $\mathrm{X}$ de la transition de phase de la pérovskite vers la phase postpérovskite, qui explique les anomalies sismiques observées à la base du manteau inférieur de la Terre [1].
Un autre exemple marquant est la synthèse in situ sous haute pression et haute température du composé $\mathrm{BC}_{2} \mathrm{~N}$ de structure cubique, et dont les propriétés mécaniques sont proches de celles du diamant [2].

On peut également noter la diversité des techniques $\mathrm{X}$ qui ont émergé au cours des deux dernières décennies. La diffraction, la diffusion inélastique, l'absorption, la diffusion Mössbauer sont autant de techniques utilisant les rayons $\mathrm{X}$, qui permettent l'étude détaillée des propriétés électroniques, magnétiques et structurales des matériaux sous conditions extrêmes.

Deux exemples de travaux récents ont été sélectionnés ici, afin d'illustrer le potentiel de ce type d'expérience. L'étude de la courbe de fusion du tantale en cellule à enclumes de diamant chauffées par laser a constitué un véritable défi expérimental et théorique et met fin à une longue controverse. Le second exemple est issu des sciences de la Terre et de l'Univers où, grâce à des mesures de densité en presse " Paris-Édimbourg ", il est proposé un scénario plausible pour expliquer l'absence de volcanisme actif à la surface de la Lune.

À travers ces deux exemples, on pourra évaluer l'intérêt des expériences in situ sous hautes pressions et températures au synchrotron. Ces méthodes, par essence multidisciplinaires, sont porteuses de découvertes importantes. 


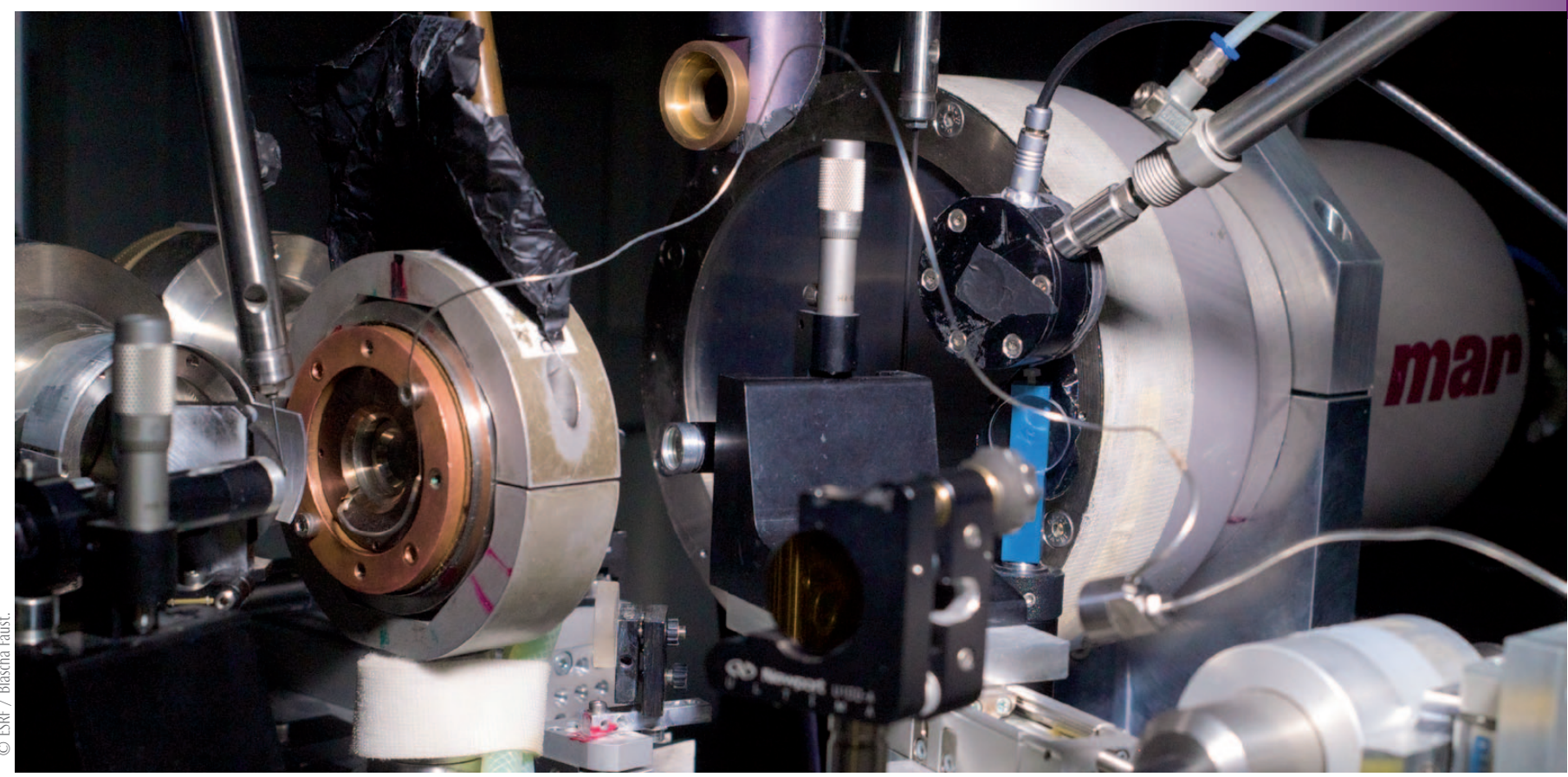

Le dispositif expérimental sur la ligne de lumière ID27 de l’ESRF, où les expériences de diffraction sont effectuées. La cellule à enclumes de diamant est à l'intérieur du cylindre de laiton au centre.

\section{Mesure de la courbe de fusion du tantale en cellule à enclumes de diamant chauffée par laser}

Dès son invention dans les années cinquante, la cellule à enclumes de diamant (CED) s'est imposée comme l'outil incontournable pour l'étude des matériaux sous hautes pressions statiques. En raison de ses propriétés physico-chimiques exceptionnelles (très grande dureté, transparence dans une large gamme de longueur d'onde, très bonne conductivité thermique), le diamant est apparu comme le matériau de confinement idéal pour les études in situ sous hautes pressions. La cellule à enclumes de diamant est un petit dispositif qui pèse environ 200 grammes et qui peut générer des pressions énormes équivalentes, voire même supérieures, à celle qui règne au centre de la Terre $(350 \mathrm{GPa}$ ou 3,5 millions d'atmosphères). De plus, en raison de ses qualités optiques dans le domaine des rayons $\mathrm{X}$, la CED est particulièrement bien adaptée aux études structurales sous hautes pressions par diffraction de rayons X. Dans ce contexte et en raison de la faible quantité de matière étudiée (quelques microgrammes), les sources de rayons $\mathrm{X}$ synchrotrons de troisième génération du type ESRF ont joué un rôle fondamental.

Une autre propriété fondamentale du diamant est sa transparence dans le domaine infrarouge, qui permet le chauffage de l'échantillon par absorption de rayonnement laser YAG ou $\mathrm{CO}_{2}$ de forte puissance. Ce système peut générer des températures supérieures à 5000 kelvins et est très bien adapté à l'étude de la courbe de fusion des métaux, dont un exemple est présenté ici.

Au cours des dix dernières années, de nombreux travaux théoriques et expérimentaux ont été consacrés à l'étude de la courbe de fusion des métaux sous haute pression. Cependant, la fusion reste une transition de phase difficile à prédire et modéliser, car elle a lieu à haute température, quand le système est éloigné de son état fondamental (à $\mathrm{T}=0 \mathrm{~K}$ ), seul état prédit de façon fiable par les calculs ab initio. Sa caractérisation expérimentale est également difficile, car il s'agit de créer et de mesurer des conditions extrêmes de pression (plusieurs millions d'atmosphères) et de température (plusieurs milliers de kelvins) et, dans le même temps, d'atteindre et de caractériser l'état d'équilibre du système.

Le cas du tantale est exemplaire des efforts, des problèmes et des controverses dans la détermination de la courbe de fusion des éléments [3]. Jusque récemment, les températures de fusion mesurées pour le tantale différaient de plusieurs milliers de kelvins dans le domaine 100-300 GPa (1-3 millions d'atmosphères). En utilisant la compression par un choc dû à l'impact d'un projectile sur l'échantillon, un point de fusion à environ $9700 \mathrm{~K}$ à $300 \mathrm{GPa}$ avait été obtenu. Mais en utilisant des cellules à enclumes de diamant chauffées par laser (CEDL), où l'échantillon comprimé entre deux diamants est chauffé par un laser infrarouge focalisé, une équipe allemande a observé visuellement des points de fusion atteignant seulement $3730 \mathrm{~K}$ à $100 \mathrm{GPa}$ [4]. Ces mesures étaient non seulement en désaccord avec les mesures par choc, mais aussi avec des prédictions théoriques pour lesquelles l'effet de la pression sur la température de fusion, $\mathrm{dT} / \mathrm{dP}$, devait être environ quatre fois plus important [5]. Il a été proposé que le changement observé en CEDL n'était pas la fusion, mais du polymorphisme [5] ou un changement des propriétés mécaniques [6].

Nous avons utilisé un nouveau diagnostic de fusion, basé sur la diffraction de rayons $\mathrm{X}$ en CEDL, pour étudier la fusion du tantale. Le phénomène de fusion s'accompagne d'une perte d'ordre à longue distance dans le matériau. Cette perte d'ordre se manifeste dans le diagramme de diffraction par la disparition des pics de Bragg et par l'apparition concomitante d'un halo diffus. L'apparition de ce halo diffus, utilisé comme critère de fusion, a été validé pour le plomb [7]. Le faisceau de rayons $\mathrm{X}$ synchrotron et les lasers sont focalisés sur un même point de l'échantillon, dont la température est 


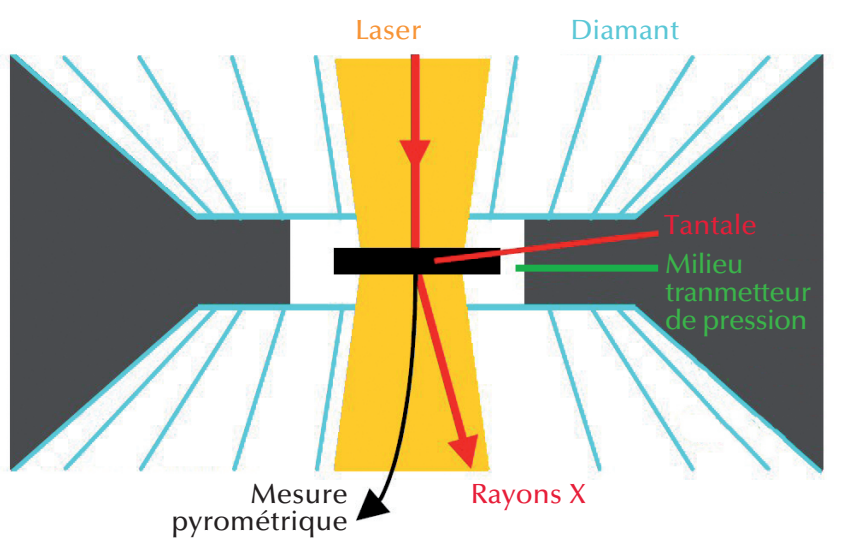

1. Schéma de la chambre de pression dans la cellule à enclumes de diamant chauffée par un faisceau laser (en jaune). Différents milieux transmetteurs de pression (sels, gaz rares, oxydes) ont été utilisés. L'échantillon est contenu dans une cavité cylindrique de 100 microns de diamètre.

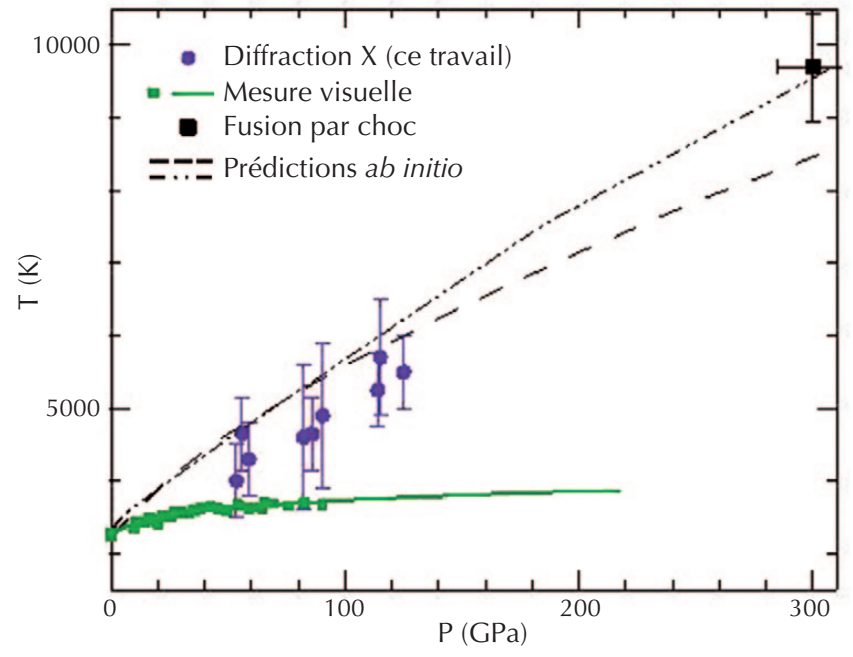

2. Courbe de fusion du tantale déterminée par différentes méthodes. À pression nulle, la température de fusion du tantale est de $3290 \mathrm{~K}$.

\section{\〉}

mesurée par pyrométrie (fig. 1). L'échantillon est entouré d'un milieu transmetteur de pression, déformable, qui permet d'obtenir des conditions de pression quasi hydrostatiques, et ce malgré la géométrie uniaxiale de la CEDL. La diffraction de rayons $\mathrm{X}$ permet de distinguer la fusion du polymorphisme, parce que ce dernier se traduit par l'apparition de nouvelles raies de Bragg; elle détecte aussi d'éventuelles réactions chimiques de l'échantillon avec son environnement au cours de la chauffe. La mesure de la dilatation thermique déduite du déplacement des raies de Bragg avec la température permet de confirmer la mesure pyrométrique.

Nos expériences ont montré qu'à haute température, le carbone, dont sont composées les enclumes de diamant de la CEDL, diffuse à travers le milieu transmetteur de pression et réagit avec le tantale pour former un carbure. Cette réaction chimique perturbe fortement l'observation de changements structuraux dans l'échantillon lors de sa chauffe. Nous avons aussi observé que la fusion du milieu transmetteur de pression peut modifier ses propriétés optiques et donc affecter les mesures pyrométriques. Ceci est un réel problème pour le tantale qui est très réfractaire, et pour lequel il est impossible de trouver un milieu transmetteur de pression qui ne fonde pas en premier. Ces difficultés peuvent expliquer les températures de fusion anormalement basses mesurées en CEDL visuellement [4].
Les mesures pyrométriques, validées par celles de dilatation thermique de l'échantillon, montrent que la température de fusion du tantale, $5500 \pm 500 \mathrm{~K}$ à $125 \mathrm{GPa}$, est bien plus élevée que ce qui avait été mesuré précédemment en CEDL. Jusqu'à ce point, le tantale ne subit aucune transformation polymorphique. La figure 2 montre que la courbe de fusion mesurée par diffraction de rayons $\mathrm{X}$ en CEDL se rapproche de la mesure par choc et est en bon accord avec les prédictions théoriques [5].

Nous pensons que cette étude a établi de nouveaux standards pour les études de physique en CED. Ces standards pourront en particulier être appliqués à l'étude du diagramme de phase du fer sous très haute pression, dont la connaissance précise est de première importance pour mieux appréhender les propriétés physico-chimiques du noyau terrestre.

\section{Étude du volcanisme lunaire en presse « Paris-Edimbourg »}

La presse Paris-Édimbourg est le fruit d'une collaboration entre deux équipes, issues de l'Université P. et M. Curie et de l'Université d'Édimbourg [8]. Ce dispositif, dont une photo est présentée sur la figure 3, a été initialement conçu pour les études in situ par diffusion de neutrons. Il s'est révélé très adapté aux mesures de diffraction et d'absorption $\mathrm{X}$ sous rayonnement synchrotron. Il permet l'étude structurale détaillée des solides et des liquides dans une large gamme de pressions et de températures ( $\mathrm{P}<15 \mathrm{GPa}$ et $300<\mathrm{T}<2500 \mathrm{~K})$. Ce dispositif présente l'avantage de pouvoir utiliser de plus gros échantillons (quelques millimètres cubes de matière) que la CED, au prix d'un domaine de pression accessible très inférieur à celui de la CED. Cet avantage est décisif, car il permet également d'insérer au sein de la cavité haute pression un micro-four résistif qui, contrairement à la CEDL, génère des conditions homogènes de température dans tout le volume de l'échantillon. Ce dernier point est crucial pour la mesure précise de la dépendance en pression et température de la densité d'un matériau.

L'exemple présenté ici est une étude récente, réalisée par une équipe de l'Université d'Amsterdam sur la ligne de lumière ID27 à l'ESRF. Elle a permis de résoudre l'énigme de l'absence de volcanisme actif à la surface de la Lune et a été publiée dans la revue Nature Geoscience [9].

Contrairement à la Terre, notre Lune n'a pas de volcanisme actif, et les traces de son activité passée datent de plusieurs milliards d'années. Pourtant, des données sismiques récentes suggèrent la présence d'une grande quantité de magma liquide à l'intérieur du satellite de la Terre. Cette proposition est confortée par les missions Apollo, qui ont permis de ramener sur Terre des échantillons de sols lunaires. L'analyse de ces échantillons représentatifs du manteau lunaire a en effet 
montré la présence de gouttelettes de verre volcanique riche en titane, provenant de véritables fontaines de lave, actives il y a plus de 3,5 milliards d'années.

Les expériences "terrestres " se sont déroulées en plusieurs étapes. En raison du caractère destructif des expériences à haute pression, il a tout d'abord fallu recréer en laboratoire des roches microscopiques du manteau lunaire. Il s'agissait de synthétiser des "copies" de celles ramenées sur Terre lors des missions Apollo. Ces roches présentent une teneur en titane plus élevée que celles du manteau terrestre.

La seconde étape a consisté à porter ces échantillons à des températures et des conditions de pression similaires à celles régnant dans le manteau lunaire, puis de mesurer leur densité par absorption de rayons $\mathrm{X}$, la diffraction $\mathrm{X}$ permettant de s'assurer de la fusion de la totalité du matériau confiné. Les échantillons ont été placés dans une enceinte de confinement en bore-époxy au centre de la presse Paris-Édimbourg (fig. 3) et ont été chauffés à l'aide d'un microfour en graphite de haute résistivité. Le mélange bore-époxy a le double avantage d'être isolant électrique et transparent aux rayons $\mathrm{X}$ de haute énergie $(\mathrm{E}>30 \mathrm{keV})$. De cette façon, il a été possible de simuler les conditions régnant dans le manteau profond de la Lune, c'est-à-dire des températures de 1800 kelvins et des pressions de 45000 atmosphères.

Une image par microsonde électronique d'un échantillon récupéré aux conditions ambiantes après compression à 10000 atmosphères et chauffage à 1854 kelvins, est présentée figure 4.

D'après les mesures de densité et les simulations numériques, il semble désormais établi que le manteau lunaire profond, même s'il est partiellement fondu, est trop dense en raison de son enrichissement en titane, pour que des panaches de matière fondue remontent en surface sous l'effet de la pression d'Archimède. Toutefois, la Lune n'ayant pas encore achevé son refroidissement et en raison de l'appauvrissement en titane du liquide par cristallisation partielle, il devrait arriver un moment dans son histoire où les conditions physico-chimiques dans le manteau lunaire devraient être telles que du magma pourrait remonter en surface. I

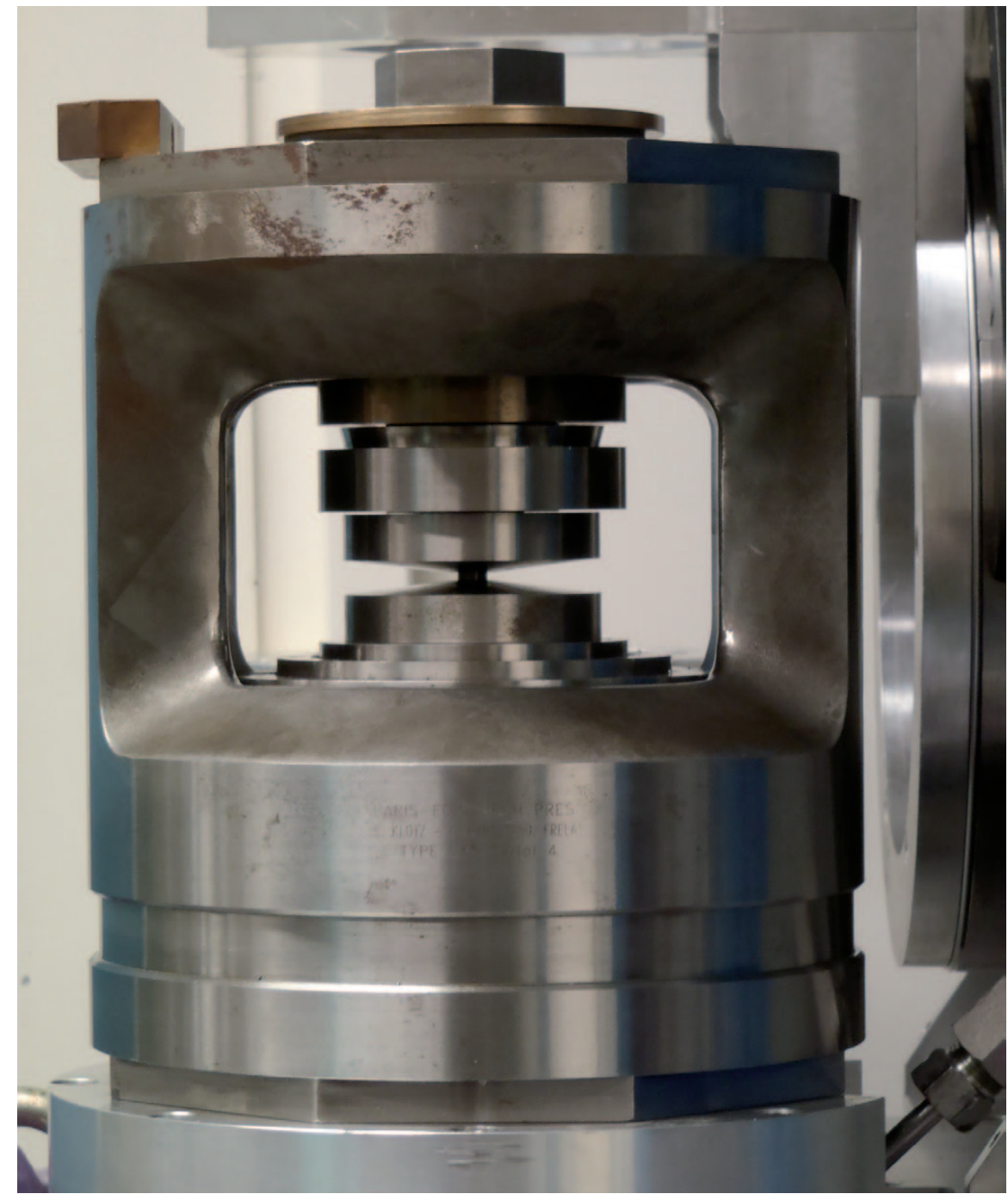

3. Photographie de la presse Paris-Édimbourg. Le joint en bore-époxy contenant l'échantillon se situe entre deux enclumes en carbure de tungstène au centre de la presse.

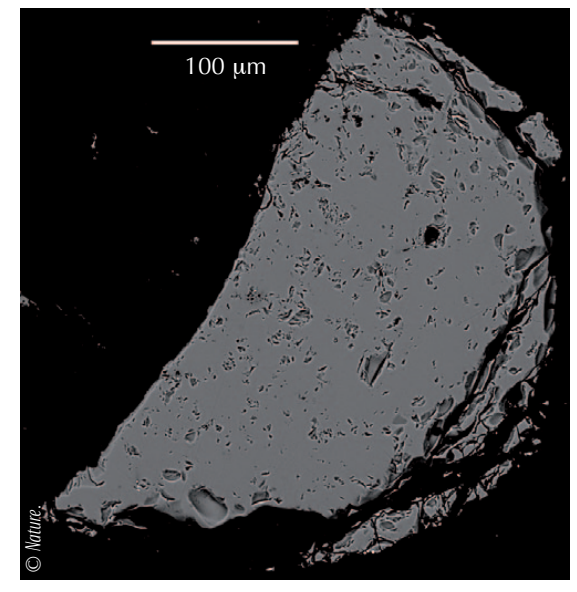

4. Image par microsonde électronique d'une copie d'échantillon de roche lunaire, après expérience aux rayons $X$.

\section{Références}

1• M. Murakami et al., Science 304 (2004) 855.

2• V.L. Solozhenko, D. Andrault, G. Fiquet, M. Mezouar, Appl. Phys. Lett. 78 (2001) 1385-1387.

3• A. Dewaele, M. Mezouar, N. Guignot et P. Loubeyre, Phys. Rev. Lett. 104 (2010) 255701.

4• D. Errandonea et al., Phys. Rev. B 63 (2001) 132104.

5• L. Burakovsky et al., Phys. Rev. Lett. 104 (2010) 255702.

6• C. J. Wu et al., Nature Mater. 8 (2009) 223.

7• A. Dewaele, M. Mezouar, N. Guignot et P. Loubeyre, Phys. Rev. B 73 (2007) 144106.

8• J. M. Besson, R. J. Nelmes, G. Hamel, J. S. Loveday, G. Weill et S. Hull, Physica B 180 \& 181 (1992) 907.

9• M. van Kan Parker et al., Nat. Geo. 5 (2012) 186. 